\title{
Regulatory network in lignin biosynthetic pathway through small RNAs in Acacia mangium: implications to the pulp and paper industry
}

\author{
Seong Siang Ong ${ }^{*}$, Ratnam Wickneswari \\ From IUFRO Tree Biotechnology Conference 2011: From Genomes to Integration and Delivery \\ Arraial d'Ajuda, Bahia, Brazil. 26 June - 2 July 2011
}

\section{Background}

Lignin, after cellulose, is the second most abundant biopolymer accounting for approximately $15-35 \%$ of the dry weight of wood. As an important component of wood, lignin is indispensable for plant structure and defense. However, it is an undesirable component in the pulp and paper industry. Removal of lignin from cellulose is a costly and environmentally hazardous process. Tremendous efforts have been devoted to understand the role of enzymes and genes controlling the amount and composition of lignin to be deposited in the cell wall. However, studies on the impact of downregulation and overexpression of monolignol biosynthesis genes in model species on lignin content, plant fitness and viability have been inconsistent. Recently, non-coding RNAs have been discovered to play an important role in regulating the monolignol biosynthesis pathway genes [1-3]. Non-coding RNAs represent an emerging class of riboregulators, which are processed to shorter miRNAs or siRNAs. The current paradigm indicated that plant system use small RNAs (miRNAs and siRNAs) as guide for post-transcriptional gene silencing and epigenetic regulation. Although miRNAs and siRNAs result from different biogenesis pathways but both interact with target transcripts for direct cleavage or translation repression, effectively shutting down that genes' functions. However, much less is known about the mechanism of gene regulation governed by these small RNAs in lignin biosynthesis pathway in $A$. mangium.

\footnotetext{
* Correspondence: ong.siang@gmail.com School of Environmental and Natural Resource Sciences, Faculty of Science and Technology, Universiti Kebangsaan Malaysia, 43600 Bangi, Selangor, Malaysia
}

(c) 2011 Ong and Wickneswari; licensee BioMed Central Ltd. This is an open access article distributed under the terms of the Creative Commons Attribution License (http://creativecommons.org/licenses/by/2.0), which permits unrestricted use, distribution, and reproduction in any medium, provided the original work is properly cited.

\section{Results}

A total of 14,582,383 reads were generated in Am54 and $10,281,313$ reads in Am48. We have identified several conserved and novel small RNAs that may serve as an

\begin{abstract}
Methods USA) following manufacturer's protocol. Thin cookies were first ground in a blender and then further ground to fine powder using mortar and pestle. Integrity of the isolated Total RNA was analyzed using Bioanalyser 2100 (Allent Technology, Palo Alto, USA) and only Total A with RIN value above 7 was selected for library construction. Library construction, sequencing and ormatics pipeline analysis was done by Gene Pool Preparation Kit (Cat. \# FC-102-1009; Illumina, San Diego, CA, USA). Illumina sequencing libraries were prepared using the 'long' IIIumina protocol according to the manufacturer's direction and two libraries were sequenced on an IIIumina GA-II following manufacturer's instructions. After masking of adapter sequences were filtered and the resulting oligos were totalled. Clustering based on relative lengths from 7 nt to $35 \mathrm{nt}$ were done using in house perl scripts. The targets were extracted by in house python script and annotated using BLAST to GO database. Only highly conserved miRNAs with strong differences in their expression level between high and low lignin secondary xylem were selected for validation using IQ5 real time - PCR technology (BioRad, Hercules, USA) (Table 1).
\end{abstract}


Table 1 Total Counts of the four selected highly conserved miRNA families isolated from secondary xylem of low lignin A. mangium 54 (Am54) and high lignin $A$. mangium 48 (Am48).

\begin{tabular}{llll}
\hline miRNA family & AM54 & Am48 & Predicted Target \\
amg-miR159 & 3158 & 1008 & MYB Trabscription Factor \\
amg-miR168 & 79040 & 33447 & Agrgonuate \\
amg-miR172 & 32743 & 18204 & APETALA 2-LIKE transcription factor \\
amg-miR394 & 2037 & 226 & F-box \\
\hline
\end{tabular}

important regulatory role during secondary wall formation. Majority of these small RNAs emerged as critical regulators for normal growth and developmental processes in A. mangium. Only a few small RNAs were postulated to play an important role during epigenetic silencing. We found that the expression level of these miRNAs belong to four different families was up regulated in tension wood (Fig. 1). Tension wood is composed almost entirely of cellulose while compression wood is rich in lignin. We will further investigate the effects of over expression of these four highly conserved miRNAs in tension wood on the expression level of monolignol biosynthetic pathway genes.

\section{Conclusions}

From the sequence results, we concluded that $A$. mangium small RNAs consist of a set of 14 highly conserved miRNAs families found in plant miRNA database, 82 novel miRNAs and a large proportion of non-conserved small RNAs with low expression levels. Out of these 14 highly conserved miRNAs families, only four miRNAs families were selected for validation in compression wood and tension wood and their total relative counts between Am54 and Am48 are shown (Table 1). Although these four miRNAs belong to different families, all of them were up regulated in tension wood,

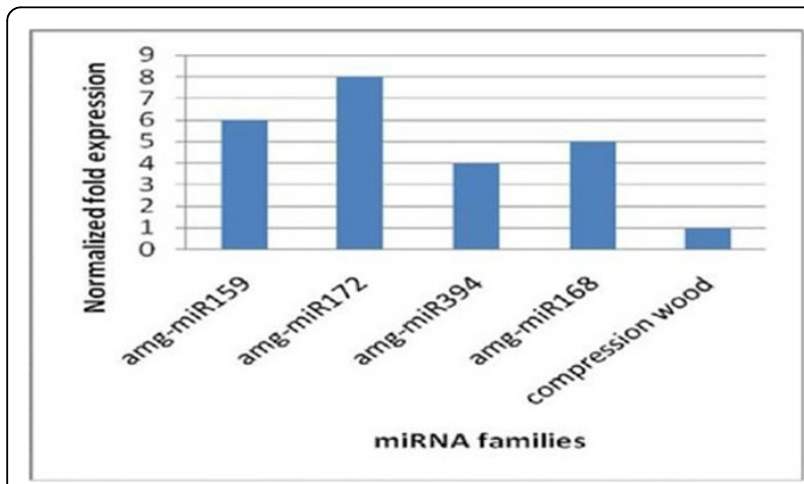

Figure 1 Relative normalized expression of four selected conserved miRNAs in secondary xylem tissue of A. mangium Tension Wood (TW) using real time PCR technology. 5.8S rRNA was selected as references genes. a region composed entirely of cellulose. The results obtained can be used to better understand the roles of small RNAs and for the development of a gene constructs for silencing of specific genes involved in monolignol biosynthesis with minimal effect on plant fitness and viability.

\section{Acknowledgements}

Special appreciation to IUFRO Tree Biotechnology Conference 2011 organizing committee for awarding Ong Seong Siang a Student Travel Grant to attend this conference. The author wish to thank Gene Pool Sequencing Centre for small RNA library preparation, sequencing and bioinformatics analysis. This work was funded by Ministry of Science, Technology and Innovation, Government of Malaysia through eScience grant 05-01-02SF1020.

\section{Published: 13 September 2011}

\section{References}

1. Baima S, Possenti M, Matteucci A, Wisman E, Altamura MM, Ruberti I, Morelli G: The Arabidopsis ATHB-8 HD-Zip protein acts as a differentiation-promoting transcription factor of the vascular meristems. Plant Physiol 2001, 126:643-655

2. Kim J, Jung JH, Reyes JL, Kim YS, Kim SY, Chung KS, Kim JA, Lee M, Lee Y, Narry Kim V, Chua NH, Park CM: MicroRNA-directed cleavage of ATHB15 mRNA regulates vascular development in Arabidopsis inflorescence stems. Plant J 2005, 42(1):84-94.

3. Lu S, Sun YH, Shi R, Clark C, Li L, Chiang VL: Novel and Mechanical Stress Responsive MicroRNAs in Populus trichocarpa That Are Absent from Arabidopsis. Plant Cell 2005, 17:2186-2203.

doi:10.1186/1753-6561-5-S7-P120

Cite this article as: Ong and Wickneswari: Regulatory network in lignin biosynthetic pathway through small RNAs in Acacia mangium: implications to the pulp and paper industry. BMC Proceedings 20115 (Suppl 7):P120.
Submit your next manuscript to BioMed Central and take full advantage of:

- Convenient online submission

- Thorough peer review

- No space constraints or color figure charges

- Immediate publication on acceptance

- Inclusion in PubMed, CAS, Scopus and Google Scholar

- Research which is freely available for redistribution 